\title{
Hardness and Approximation of Octilinear Steiner Trees ${ }^{\star}$
}

\author{
Matthias Müller-Hannemann ${ }^{1}$ and Anna Schulze ${ }^{2}$ \\ 1 Technische Universität Darmstadt, Department of Computer Science, \\ Hochschulstraße 10, 64289 Darmstadt, Germany, \\ muellerh@algo.informatik.tu-darmstadt .de, \\ http://www . algo.informatik.tu-darmstadt.de \\ 2 Zentrum für Angewandte Informatik Köln, Weyertal 80, 50931 Köln, Germany \\ schulze@zpr.uni-koeln.de
}

\begin{abstract}
Given a point set $K$ of terminals in the plane, the octilinear Steiner tree problem is to find a shortest tree that interconnects all terminals and edges run either in horizontal, vertical, or $\pm 45^{\circ}$ diagonal direction. This problem is fundamental for the novel octilinear routing paradigm in VLSI design, the socalled X-architecture.

As the related rectilinear and the Euclidian Steiner tree problem are well-known to be NP-hard, the same was widely believed for the octilinear Steiner tree problem but left open for quite some time. In this paper, we prove the NPcompleteness of the decision version of the octilinear Steiner tree problem.

We also show how to reduce the octilinear Steiner tree problem to the Steiner tree problem in graphs of polynomial size with the following approximation guarantee. We construct a graph of size $O\left(\frac{n^{2}}{\varepsilon^{2}}\right)$ which contains a $(1+\varepsilon)$-approximation of a minimum octilinear Steiner tree for every $\varepsilon>0$ and $n=|K|$. Hence, we can apply any $\alpha$-approximation algorithm for the Steiner tree problem in graphs (the currently best known bound is $\alpha \approx 1.55)$ and achieve an $(\alpha+\varepsilon)$ - approximation bound for the octilinear Steiner tree problem. This approximation guarantee also holds for the more difficult case where the Steiner tree has to avoid blockages (obstacles bounded by octilinear polygons).
\end{abstract}

Keywords: octilinear Steiner trees, NP-completeness, VLSI design, approximation algorithms, blockages

\section{Introduction}

Background and motivation. In recent years there has been strong and growing interest in a new routing paradigm in VLSI design: octilinear routing, the so-called Xarchitecture $[\mathrm{X}]$. In addition to vertical and horizontal wires, octilinear routing allows wiring in 45- and 135-degree directions. Compared to traditional and state-of-the-art rectilinear (Manhattan) routing, such a technology promises clear advantages in wire length and via reduction. As a consequence a significant chip performance improvement and power reduction can be obtained (with estimations being in the range of $10 \%$ to $20 \%$ improvement) [Tei02,PWZ04]. To enable such a technology, novel algorithmic

\footnotetext{
* An extended abstract of this paper appears in Proceedings of the 16th Annual International Symposium on Algorithms and Computation 2005 (ISAAC 2005), Sanya, Hainan, China, LNCS, Springer.
} 
approaches for the construction of octilinear Steiner trees are needed. An octilinear Steiner tree is a tree that interconnects a set of points (terminals) in the plane with minimum length such that every line segment uses one of the four given orientations.

Even more general routing architectures are obtained if a fixed set of uniformly oriented directions is allowed. For an integral parameter $\lambda \geq 2$, consecutive orientations are separated by a fixed angle of $\pi / \lambda$. A $\lambda$-geometry is a routing environment in which every line segment uses one of the given orientations. Manhattan routing can then be seen as the special case $\lambda=2$ and the $\mathrm{X}$-architecture as the case $\lambda=4$. A Steiner minimum tree in a $\lambda$-geometry is called $\lambda$-SMT.

In this paper we focus on the octilinear case (although most of our results can be generalized to arbitrary $\lambda \geq 2$ ). We study approximation algorithms for the octilinear Steiner tree problem with and without obstacles. The rectilinear and the Euclidean Steiner tree problem have been shown to be NP-hard in [GJ77] and [GGJ77], respectively. It is widely believed that the Steiner tree problem is NP-hard for every fixed $\lambda$ (although this question seems to be open [Cou03]). Here, we present the proof that the octilinear Steiner tree problem is indeed NP-hard.

Blockages. In VLSI design routing is often restricted by the presence of blockages (or obstacles) which exclude certain areas for possible interconnections. Throughout this paper, an obstacle is a connected region in the plane bounded by a simple polygon. For a given set of obstacles $\mathcal{O}$ we require that the obstacles be disjoint, except for possibly a finite number of common points. If all boundary edges of an obstacle are rectilinear, we call such an obstacle a rectilinear obstacle. Analogously, if all obstacle edges lie within the 4-geometry, such an obstacle is called octilinear obstacle. In practice, obstacles are caused by preplaced macros or other circuits and can be assumed to be rectilinear.

Previous work. It is fairly easy to see that the approximation schemes of Arora [Aro98] and Mitchell [Mit99] are also applicable to the octilinear Steiner tree problem (without obstacles). Rao and Smith [RS98] even improved the running time of a $(1+\varepsilon)$ approximation to $O(n \log n)$. Unfortunately, the hidden constants of the asymptotic running time grow exponentially depending on $\varepsilon$. Hence, in spite of its theoretical importance, the practical value of these approximation schemes might be limited. Heuristics have been proposed by Kahng et al. [KMZ03] and Zhu et al. [ZZJ $\left.{ }^{+} 04\right]$.

Exact approaches to the octilinear Steiner tree problem have been developed by Nielsen, Winter and Zachariasen [NWZ02] and Coulston [Cou03]. Nielsen et al. report the exact solution to a large instance with 10000 terminals within two days of computation time. However, we are not aware of exact approaches or approximations in the presences of obstacles.

Transformation to Steiner tree problem in graphs. For rectilinear Steiner tree problems for point sets in the plane the most successful approaches are based on transformations to the related Steiner tree problem in graphs. Given a connected graph $G=(V, E)$, a length function $\ell$, and a set of terminals $S \subseteq V$, a Steiner tree is a tree of $G$ containing all vertices of $S$. A Steiner tree $T$ is a Steiner minimum tree of $G$ if the length of $T$ is minimum among all Steiner trees. The best available approximation guarantee for the Steiner problem in general graphs is $\alpha=1+\frac{\ln 3}{2} \approx 1.55$, obtained by Robins and Zelikovsky [RZ00].

Given a finite point set $K$ in the plane, the so-called Hanan grid [Han66] is obtained by constructing a vertical and a horizontal line through each point of $K$. The impor- 
tance of the Hanan grid lies in the fact that it contains a rectilinear Steiner minimum tree. An implementation by Althaus, Polzin and Daneshmand [APD03] is the currently strongest available exact approach for both the Steiner tree problem in graphs and the rectilinear Steiner tree problem.

$\mathrm{Du}$ and Hwang [DH92] generalized the Hanan grid construction to $\lambda$-geometries. They define grids $G_{k}(K)$ recursively in the following way. For an instance with point set $K, G_{0}(K)=K$. The grid $G_{1}(K)$ is constructed by taking $\lambda$ (infinite) lines with orientations $\pi / \lambda, 2 \pi / \lambda, \ldots,(\lambda-1) \pi / \lambda, \pi$ for each point of $K$. The $k$-th grid $G_{k}(K)$ for $k>1$ is constructed from the $(k-1)$-th grid by adding for each intersection point $x$ of lines in $G_{k-1}(K)$ additional lines through $x$ with orientations $\pi / \lambda, 2 \pi / \lambda, \ldots,(\lambda-$ 1) $\pi / \lambda, \pi$. Lee and Shen [LS96] showed that for every instance of the Steiner tree problem in a $\lambda$-geometry with $\lambda \in \mathbb{N}_{\geq 2}$, there is a minimum $\lambda$-Steiner tree which is contained in $G_{n-2}(K)$. This result has been strengthened for octilinear Steiner trees by Lin and Xue [LX00]. They showed that a minimum octilinear Steiner tree is already contained in the grid $G_{([2 n / 3\rceil-1)}(K)$. Unfortunately, the graph $G_{k}(K)$ has $O\left(n^{2^{k}}\right)$ vertices and edges. Hence, in general an optimal solution requires an exponentially large graph.

It is therefore an interesting open question which approximation guarantee for the octilinear (or $\lambda$-) Steiner tree problem can be achieved if one works with a graph $G_{k}(K)$ for some fixed constant $k$.

Some partial answers to this question are obvious. Since $G_{1}(K)$ contains a shortest path between any pair of terminals it also contains the solution obtained from the minimum spanning tree heuristic to approximate the Steiner minimum tree. Therefore, its performance guarantee cannot be worse than the Steiner ratio. The Steiner ratio is the smallest upper bound on the ratio between the length of a minimum spanning tree and the length of a Steiner minimum tree. The Steiner ratio in the octilinear case is $\frac{4}{2+\sqrt{2}}\left[\right.$ Koh95,She97]. This implies that $G_{1}(K)$ contains a solution which is not more than about $17.15 \%$ above the minimum. In this paper, we show how to modify $G_{1}(K)$ so that we can derive stronger approximation guarantees. For any $k \in \mathbb{N}$ we construct a graph of size $O\left(k^{2} n^{2}\right)$, which contains a $\left(1+\frac{1}{k}\right)$-approximation.

Our contribution. We summarize the main results of this paper:

- We establish the NP-completeness of the decision version of the octilinear Steiner tree problem.

- For a given set of $n$ terminals in the plane and for every $\varepsilon>0$ we construct a graph of size $O\left(\frac{n^{2}}{\varepsilon^{2}}\right)$ which contains a $(1+\varepsilon)$-approximation of a minimum octilinear Steiner tree.

- If $\alpha$ denotes the approximation guarantee of an algorithm for the Steiner tree problem in graphs, then we achieve an $(\alpha+\varepsilon)$-approximation guarantee for the octilinear Steiner tree problem with or without blockages.

Overview. The remaining part of the paper is organized as follows. In Section 2 we state some basic definitions and facts about octilinear Steiner trees. Afterwards, we present our NP-completeness proof. Then, in Section 4, we derive our approximation for the case without blockages. Finally, we briefly point out why the same method also works in the presence of blockages. 


\section{Basic Definitions and Facts for Octilinear Steiner Trees}

In this section we recall some basic definitions and known facts about optimal octilinear Steiner trees which will be used in the later analysis of our approach, see for example [LS96].

Property 1. The number of Steiner points for a Steiner tree on $n$ terminals is at most $n-2$.

Property 2. The degree of any Steiner point is either three or four. There exists a Steiner minimum tree such that every degree-4 Steiner point is adjacent to four terminals which form a cross.

Property 3. There exists an octilinear Steiner minimum tree $T_{\text {opt }}$ such that the three angles around a degree-3 Steiner point are $\frac{\pi}{2}, \frac{3 \pi}{4}, \frac{3 \pi}{4}$ (in some order).

A Steiner tree is a full Steiner tree if all its terminals are leaves. Any Steiner tree can be decomposed into its full components.

Property 4 ([BTW00]). Given a set of terminals $K$ such that every octilinear Steiner minimum tree is a full Steiner tree, there is an octilinear Steiner minimum tree $T_{\text {opt }}$ such that all but at most one edge are straight edges. The latter one may bend once.

Property 5 ([BTW00]). A non-straight edge bends at its corner point by an angle of $\frac{3 \pi}{4}$. Let $p s$ be a non-straight edge in an octilinear Steiner minimum tree such that $s$ is a Steiner point. Let $c$ be the corner point of $p s$, and let $q$ and $r$ be the other two vertices adjacent to $s$. If $c$ lies on the same side of the line through $p s$ as $q$, then $\angle c s q=\frac{\pi}{2}$ and $\angle c s r=\frac{3 \pi}{4}$.

\section{NP-Completeness of the Octilinear Steiner Tree Problem}

In this section we prove that the decision version of the octilinear Steiner tree problem is NP-complete. We have the following decision problem:

Problem: Octilinear Steiner tree decision problem

Instance: A set $K$ of terminals with integral coordinates in the plane and a number $L \in \mathbb{N}$.

Task: Is there an octilinear Steiner tree $T$ with $l(T) \leq L$ ?

At a first glance, it might not even be clear whether the decision version of the octilinear Steiner tree problem belongs to the class NP, since the distance between terminals and/or Steiner points may be irrational. In sharp contrast to the Euclidian version where this question is still open, we can prove membership in NP for the octilinear case.

Lemma 1. The decision version of the octilinear Steiner tree problem belongs to the class NP. 
Proof. To show membership in NP, we need a certificate which a nondeterministic algorithm can guess and we can verify in polynomial time. In this case, an appropriate certificate is the topology of an optimal tree $T$. (In this context, topology means a graph which includes the terminals and Steiner points as vertices and specifies the connections between these vertices as edges.)

From the topology of a tree, one can compute an optimal realization in the plane in linear time [BTWZ02]. Using our assumption that all terminals have integral coordinates, it is easy to see that all Steiner points have rational coordinates (namely by traversing the tree from its leaves). Moreover, the encoding length of each Steiner point does not become too large. If $s$ denotes the maximum number of bits to store the coordinates of some input terminal, then the encoding length of each Steiner point is upper bounded by $O(s+|K|)$. This follows from the fact that all line segments are either horizontal, vertical or have slopes \pm 1 . Hence, we can express the length of each tree edge $e$ by $\ell(e)=a_{e}+b_{e} \cdot \sqrt{2}$, where $a_{e}$ and $b_{e}$ are rational numbers of polynomial size with respect to the input. The length of the Steiner tree can be evaluated as $\ell(T)=a+b \sqrt{2}$, where $a=\sum_{e \in T} a_{e}$ and $b=\sum_{e \in T} b_{e}$. Hence, $\ell(T) \leq L$ if and only if $2 \leq\left(\frac{L-a}{b}\right)^{2}$.

Theorem 1. The decision version of the octilinear Steiner tree problem is NP-complete.

To prove this theorem, we basically use the same idea of a reduction as Garey, Graham and Johnson [GGJ77] provided in their hardness proof for the Euclidean Steiner tree problem. The main difference lies in the proof that this reduction is correct.

Before we go into the details, we briefly sketch the construction and point out the technical differences to the Euclidean case in the proof of its correctness. The problem we reduce to the octilinear Steiner tree decision problem is that of EXACT COVER BY 3-SETS:

Problem: Exact cover by 3 -sets

Instance: A family $\mathcal{F}=\left\{F_{1}, F_{2}, \ldots, F_{t}\right\}$ of 3 -element subsets of a set $F$ of $3 n$ elements. Without loss of generality let $F=\{1,2, \ldots, 3 n\}$.

Task: Is there a subfamily $\mathcal{F}^{\prime} \subseteq \mathcal{F}$ such that distinct elements of $\mathcal{F}^{\prime}$ are disjoint and $\bigcup_{F_{i} \in \mathcal{F}^{\prime}} F_{i}=F$ ?

The 3-dimensional matching problem shown to be NP-complete in [Kar72] is a special case of the problem EXACT COVER BY 3-SETS. Therefore, EXACT COVER BY 3-SETS is also NP-complete.

The main difficulty in the NP-completeness proof is due to the problem that it is hard to argue about the optimality of some Steiner tree unless we have very few terminals or very restricted locations for them. Hence, a reduction requires gadgets of very small size. As gadgets have to be combined with each other, we would like to have that the possible configurations of optimal Steiner trees can easily be enumerated for each subset of terminals contained in the gadget. The overall configuration is composed by gadgets of rows of terminals which meet in "triangles" or "squares", see Fig. 1. Gadgets of these types are connected by long rows of terminals. Hence, they are placed far enough from each other, so that they do not directly mutually interact. In contrast, adjacent terminals of the same row are relatively near to each other, they have a distance of at most $1 / 10$. This has the important effect that there is a spanning tree such that each edge has length at most 1 . For a tree $T$ denote by $m(T)$ the maximum 


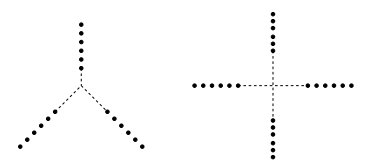

Fig. 1. Basic gadgets: terminals are dots; possible locations of Steiner points which are not excluded by probes are displayed with dashed lines.

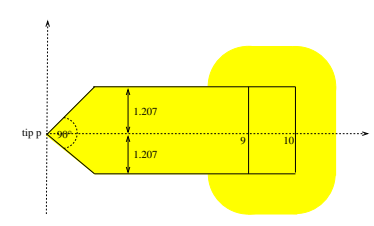

Fig. 2. The region of a probe $P$ with tip $p$ is the shaded area.

length of some edge in $T$. From this property, we can conclude that also every edge in an optimum Steiner tree $T_{\text {opt }}$ cannot be longer than 1 , that is $m\left(T_{\text {opt }}\right) \leq 1$. The composition of gadgets is the same as in the proof for the Euclidian case [GGJ77].

A fundamental idea in the proof is to restrict the possible locations of Steiner points to certain so-called active regions. To exclude other regions for Steiner points, we use a discrete version of the concept of so-called probes which are regions with a geometric shape as in Fig. 2. The central node of a probe is called the tip of the probe. We say that a probe $P$ is valid if it is rotated by an integral multiple of $\pi / 4$ around its tip. In the Euclidean case, probes can be rotated by any angle and have a slightly different shape. The proof of the following lemma requires some preparation and will appear in Subsection 3.3

Lemma 2. If $p$ is a Steiner point of an octilinear Steiner minimum tree with $m\left(T_{\text {opt }}\right) \leq$ 1 , then there must be at least one terminal located inside every valid probe $P$ with tip p.

These properties will enable us to conclude that any two terminals which are at most 1/10 apart from each other must be connected by an edge in any minimum Steiner tree. Hence, the whole combinatorial difficulty lies in the problem how to connect these components to a tree for the overall configuration of terminals.

Given a configuration of terminals which encode an instance $\mathcal{F}$ of EXACT COVER BY 3-SETS, one has to show that an optimal Steiner tree for this configuration does not exceed a value $L=L(\mathcal{F})$ if and only if there is an exact cover. The value of $L$ depends only on parameters $t$ and $n$ of $\mathcal{F}$. Again one can argue along the lines of the proof in [GGJ77]. However, compared to the Euclidian case, optimal subtrees inside active regions have different lengths. Therefore, it is crucial to note that certain inequalities about the relative lengths of such subtrees remain valid.

The formal proof of Theorem 1 is structured as follows. In Section 3.1 we first introduce the gadgets used in the reduction from EXACT COVER BY 3-SETS. Afterwards, in Sections 3.2 and 3.3 we obtain several auxiliary and geometrical results which lead to the "probing lemma" (Lemma 2). From the probing lemma we can deduce several properties of the structure of optimal Steiner trees for configurations of terminals as they appear in our reduction (Section 3.4). Finally, the proof is completed by showing that the configuration of an instance of EXACT COVER BY 3-SETS has an optimal Steiner tree of a certain length if we have a YES-instance and is strictly larger otherwise.

\subsection{Formal Definition of Gadgets}

In this section we describe a configuration $K=K(\mathcal{F})$ of terminals in the plane which transforms an instance of EXACT COVER BY 3-SETS into an instance of the oc- 


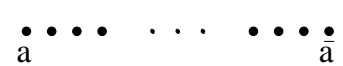

(a)

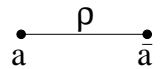

(b)

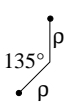

(a)

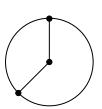

(b)

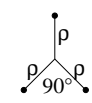

(c)

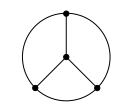

(d)

Fig. 3. A standard row $\rho$.

Fig. 4. An angle $A$ and junction $J$.

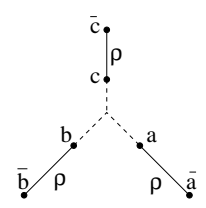

(a)

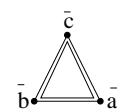

(b)

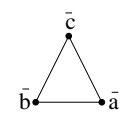

(c)

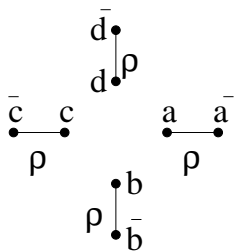

(a)

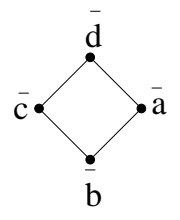

(b)

Fig. 5. A triangle $R(\epsilon)$.

tilinear Steiner tree problem. We assume both $n$ and $t$ exceed 1 , since otherwise the problem EXACT COVER BY 3-SETS would be trivial.

The set $K$ is constructed by different components. A standard row, shown in Fig. 3, consists of 100 equally spaced terminals lying in a straight line with adjacent terminals separated by a distance of $1 / 10$. The terminals $a$ and $\bar{a}$ are called the endpoints of the row. We denote a standard row schematically as in Fig. 3(b).

Next we combine standard rows to define other components. An angle $A$ consists of two standard rows with a common endpoint. The two rows are embedded on lines which meet at an angle of $135^{\circ}$. See Fig. 4(a) and Fig. 4(b) for the schematic representation. A junction $J$ consists of three normal rows with a common endpoint. One of the three rows meets the other two at $135^{\circ}$. The other two meet at $90^{\circ}$. See Fig. 4(c) and Fig. 4(d) for the schematic representation.

In Fig. 5(a), we show a configuration $R(\epsilon)$ composed of three standard rows. The endpoints $a, b$ and $c$, called the active terminals of $R(\epsilon)$, are the vertices of an isosceles triangle. The length of each side is $1-\epsilon$ in the octilinear metric for some $\epsilon, 0 \leq \epsilon<$ $1 / 200$, to be specified later. The three standard rows radiate out from the three vertices and lie outside the triangle. The row beginning at the common endpoint of the two sides of the same length lies on a vertical line. The extensions of the two other rows meet the vertical row each at $135^{\circ}$. When $\epsilon>0, R(\epsilon)$ is called a small triangle and is denoted schematically as in Fig. 5(b). When $\epsilon=0, R(0)=R$ is called a standard triangle and is denoted schematically as in Fig. 5(c).

In Fig. 6(a), we show a configuration $Q(\epsilon)$ composed of four standard rows. The endpoints $a, b, c$, and $d$ are the active terminals of $Q(\epsilon)$ and form the vertices of a square of side length $1-\epsilon, 0 \leq \epsilon<1 / 200$. The standard rows lie on the extended diagonals of the square. We call $Q(\epsilon)$ a square and denote it schematically as in Fig. $6(\mathrm{c})$. We call the regions lying inside a triangle $D(\epsilon)$ or inside a square $Q(\epsilon)$ active regions.

Finally, we form a fundamental configuration, called crossover $C$, shown in Fig. 7, by combining some of the previous configurations. A crossover $C$ consists of two normal 


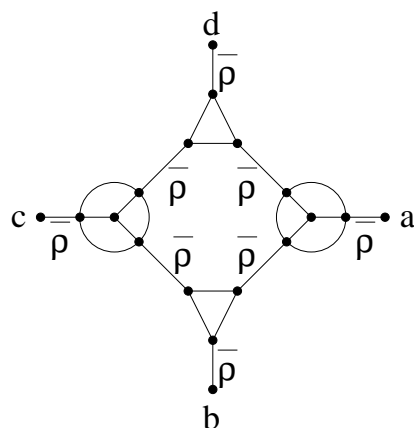

(a)<smiles>C1CCCCC1</smiles>

(b)

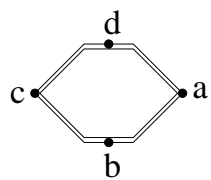

(c)

Fig. 7. A crossover $C$.

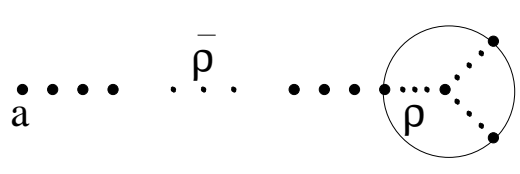

Fig. 8. A typical connection.

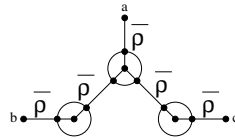

(a) (b) (c)

Fig. 9. A terminator $\Omega$.

triangles $R$, two junctions $J$ and a number of rows of terminals, denoted by $\bar{\rho}$ used to interconnect these components. Each row $\bar{\rho}$ is called long row and consists of at least 1000 terminals lying on a straight line with distances between consecutive terminals ranging between $1 / 11$ and $1 / 10$. Both endpoints of a long row are endpoints of standard rows of components which are interconnected by the long row. Two rows with the same endpoint are collinear, see Fig. 8 for a typical connection.

The exact positions of terminals in each row $\bar{\rho}$ are chosen so that the component $C$ shown in Fig. 7(a) is geometrically realizable. For the schematic representation see Fig. 7(b). If $C$ is constructed using a small triangle $R(\epsilon)$ in place of the upper standard triangle, we call the crossover small crossover $C(\epsilon)$ and represent it schematically as in Fig. 7(c).

Two angles $A$, a junction $J$ and five long rows $\bar{\rho}$ are connected to a terminator, shown in Fig. 9(a). A terminator is denoted as downward terminator or as upward terminator respectively, depending on whether the terminal $a$ lies under or upper the other terminals of the terminator. The schematic representation is shown in Fig. 9(b) and (c).

With these components we construct the set $K(\mathcal{F})$ of terminals for the octilinear Steiner tree problem. It will be formed by connecting junctions, squares and terminators by long rows $\bar{\rho}$. $K(\mathcal{F})$ contains a chain of $t+1$ upward terminators $\Omega_{k}, 0 \leq k \leq t$, connected by long rows. Each upward terminator $\Omega_{i}, 1 \leq k \leq t$, is connected with a square $Q_{k}$ that again is connected with two upward terminators $\Omega_{k}^{\prime}$ and $\Omega_{k}^{\prime \prime}$.

We associate with each square $Q_{k}$ the subset $F_{k}=\left\{a_{k}, b_{k}, c_{k}\right\} \in \mathcal{F}$. From each square $Q_{k}$, a chain of crossovers $C_{k}(i)$ for $0 \leq i<a_{k}$ connected by long rows emanates 


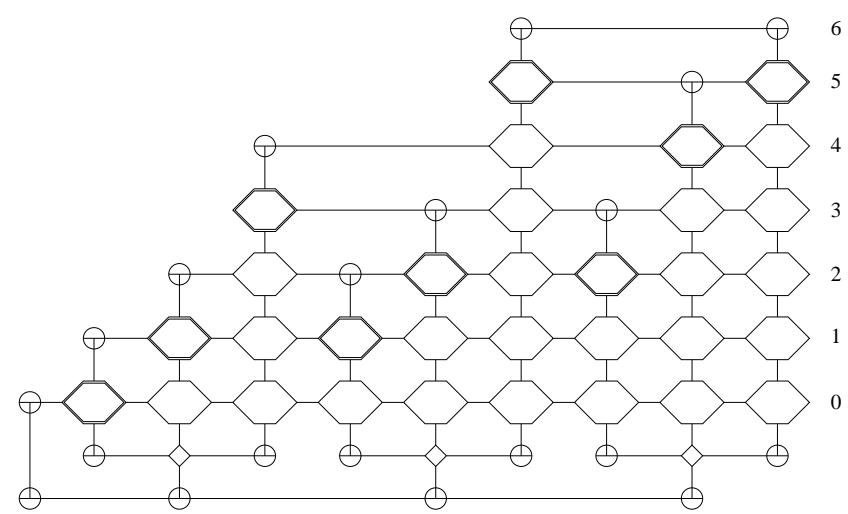

Fig. 10. Example for the overall construction of $K\left(\mathcal{F}_{0}\right)$.

in upward direction. The crossover $C_{k}\left(a_{k}-1\right)$ is a small crossover $C(\epsilon)$. The other crossovers $C_{k}(i)$ are standard crossovers. A downward terminator $\bar{\Omega}_{k}$ lies above $C_{k}\left(a_{k}-\right.$ 1). Similarly, above $\Omega_{k}^{\prime}$ and $\Omega_{k}^{\prime \prime}$ there are chains of crossovers $C_{k}^{\prime}(i), 0 \leq i<b_{k}$, and $C_{k}^{\prime \prime}(j), 0 \leq j<c_{k}$, respectively, with $C_{k}^{\prime}\left(b_{k}-1\right)$ and $C_{k}^{\prime \prime}\left(c_{k}-1\right)$ being small crossovers each of the two connected to a downward terminator $\bar{\Omega}_{k}^{\prime}$ and $\bar{\Omega}_{k}^{\prime \prime}$. All the $C_{k}(i), C_{k}^{\prime}(i)$ and $C_{k}^{\prime \prime}(i)$ lie at the same horizontal level, called the $i t h$ level, for $1 \leq k \leq t$. Therefore, $\bar{\Omega}_{k}$ lies at the $a_{k}$ th level, $\bar{\Omega}_{k}^{\prime}$ and $\bar{\Omega}_{k}^{\prime \prime}$ at the $b_{k}$ th and $c_{k}$ th level, respectively. A downward terminator $\Omega_{0}^{\prime}$ at level zero is connected with the upward terminator $\Omega_{0}$ below it. Finally, all components at the same level are connected by a chain of long rows.

As an example, we show in Fig. 10 a schematically representation of $K(\mathcal{F})$ for the family $\mathcal{F}_{0}=\{\{1,2,4\},\{2,3,6\},\{3,5,6\}\}$. The interconnecting lines represent long rows, chosen geometrically possible. The square on the left in Fig. 10 represents the set $\{1,2,4\}$, the middle the set $\{2,3,6\}$ and the square on the right the set $\{3,5,6\}$.

In general, $K(\mathcal{F})$ contains $6 t+2$ terminators, $t$ squares, $3 t$ small crossovers and at most $9 n t-3 t$ standard crossovers. The various components can be placed so that the encoding length of $K(\mathcal{F})$ is bounded by a polynomial in $n$ and $t$.

\subsection{Auxiliary Results}

Throughout this section, let $K$ be a set of terminals in the plane. In a spanning or a Steiner tree $T$ for $K$ the unique path between two terminals $s, t \in K$ will be denoted by $P_{T}(s, t)$. The maximum length of an edge in a tree $T$ or in a path $P_{T}(s, t)$ will be denoted by $m(T)$ and $m\left(P_{T}(s, t)\right)$, respectively. We denote by $T^{\text {span }}$ a spanning tree for $K$, by $T$ an octilinear Steiner tree for $K$, and by $T_{o p t}^{\text {span }}$ and $T_{o p t}$ a minimum spanning tree and an octilinear Steiner minimum tree for $K$, respectively. The following result appears in [GGJ77].

Lemma 3. For any set $K$ and terminals $s, t \in K$,

$$
m\left(P_{T^{\text {span }}}(s, t)\right) \geq m\left(P_{T_{o p t}^{s p a n}}(s, t)\right) \geq m\left(P_{T_{o p t}}(s, t)\right) .
$$

Proof. Suppose there exists a spanning tree $T^{\text {span }}$ of $K$ and $s, t \in K$ with $m\left(P_{T^{s p a n}}(s, t)\right)$ $<m\left(P_{T_{o p t}^{s p a n}}(s, t)\right)$. This implies that there exists an edge $e$ with $l(e)=m\left(P_{T_{o p t}^{s p a n}}^{\text {span }}(s, t)\right)$ 


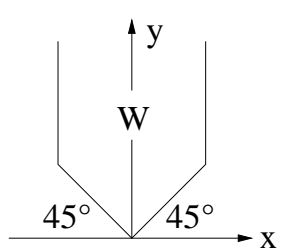

Fig. 11. The semi-infinite strip $W$.

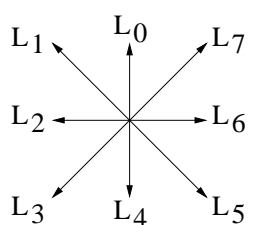

Fig. 12. Possible directions of edges in a Steiner minimum tree.

in $P_{T_{\text {opt }}^{\text {span }}}(s, t)$ which is longer than every edge of $P_{T^{\text {span }}}(s, t)$. If we delete this edge $e$ from $T_{o p t}^{\text {span }}$, the resulting graph consists of exactly two connected components. The addition of some edge $e^{\prime}$ of $P_{T}$ span $(s, t)$ must rejoin these two components, forming a spanning tree $T^{\prime}$ span for $K$ with

$$
\begin{aligned}
l\left(T^{\prime \text { span }}\right) & =l\left(T_{\text {opt }}^{\text {span }}\right)-l(e)+l\left(e^{\prime}\right) \\
& \leq l\left(T_{\text {opt }}^{\text {span }}\right)-m\left(P_{T_{\text {opt }}^{\text {span }}}(s, t)\right)+m\left(P_{T^{\text {span }}}(s, t)\right) \\
& <l\left(T_{\text {opt }}^{\text {span }}\right)
\end{aligned}
$$

which contradicts the definition of a minimal spanning tree $T_{o p t}^{\text {span }}$ for $K$. This proves the first inequality in (1). The second inequality follows similarly.

\subsection{Geometrical Results}

In this section we prove some geometrical results of octilinear Steiner minimum trees. We restrict our attention to full components. By Property 4, we may assume that a full component of an octilinear Steiner minimum tree $T_{\text {opt }}$ has at most one bending edge. Define the semi-infinite strip $W$, shown in Fig. 11 by

$$
W=\left\{(x, y)|| x\left|\leq \frac{1+\sqrt{2}}{2}, y \geq\right| x \mid\right\} .
$$

Lemma 4. Let $T_{\text {opt }}$ a octilinear Steiner minimum tree for $K$ with $m\left(T_{\text {opt }}\right) \leq 1$ and suppose $s_{0}=(0,0)$ is a Steiner point of $T_{\text {opt }}$. Then for some terminal $x$ of $K$, the whole path from $s_{0}$ to $x$ in $T_{\text {opt }}$ will be contained in $W$, i.e.,

$$
P_{T_{\text {opt }}}\left(s_{0}, x\right) \subseteq W .
$$

Proof. We denote the possible directions of edges incident to $s_{0}$ by $L_{0}, L_{1}, \ldots, L_{7}$ in counter-clockwise order. Lines in direction $L_{0}$ meet the positive $x$-axis at $90^{\circ}$. See also Fig. 12.

We partition $W$ into three sets. $W_{1}$ is the set of points in $W$ which would be moved out of $W$ if they were translated in the direction of $L_{1}$ by 1 unit. $W_{2}$ is the set of points in $W$ which would be moved out of $W$ if they were translated in the direction of $L_{7}$ by 1 unit. $W_{3}$ is the set of point in $W$ which would remain in $W$ if translated by 1 unit in the direction of either $L_{1}$ or $L_{7}$. See Fig. 13 for an illustration. 
Therefore, the three sets are defined as follows:

$$
\begin{aligned}
& W_{1}=\{(x, y) \in W \mid x<-1 / 2\} \\
& W_{2}=\{(x, y) \in W \mid x>1 / 2\} \\
& W_{3}=W-\left(W_{1} \cup W_{2}\right) .
\end{aligned}
$$

Observe for considerations to be done later that the width of $W_{3}$ is 1 .

We show how to choose a path $\left(\left\{s_{0}, s_{1}\right\},\left\{s_{1}, s_{2}\right\}, \ldots,\left\{s_{t-1}, s_{t}\right\}\right)$ of $T_{o p t}$ within $W$ that ends in a terminal $s_{t}$. The first point of the path is the Steiner point $s_{0}=(0,0)$. Suppose the last point of the path we have chosen is the point $s_{i}$ which is not a terminal. We use the following rules for choosing the next Steiner point $s_{i+1} \neq s_{i-1}$ and the edge $\left\{s_{i}, s_{i+1}\right\}$ of the path:

If $s_{i} \in W_{1}$, choose $\left\{s_{i}, s_{i+1}\right\}$ so that $\left\{s_{i}, s_{i+1}\right\}$ is an edge of $T_{\text {opt }}$ with the direction $L_{0}$, $L_{6}$ or $L_{7}$.

If $s_{i} \in W_{2}$, choose $\left\{s_{i}, s_{i+1}\right\}$ so that $\left\{s_{i}, s_{i+1}\right\}$ is an edge of $T_{\text {opt }}$ with the direction $L_{0}$, $L_{1}$ or $L_{2}$.

If $s_{i} \in W_{3}$, choose $\left\{s_{i}, s_{i+1}\right\}$ so that $\left\{s_{i}, s_{i+1}\right\}$ is an edge of $T_{\text {opt }}$ with the direction $L_{0}$, $L_{1}$ or $L_{7}$. If the edge $\left\{s_{i}, s_{i+1}\right\}$ has direction $L_{1}$ or $L_{7}$ and bends so that the other part of the edge has direction $L_{2}$ or $L_{6}$ respectively (by Property 5 ), choose $\left\{s_{i}, s_{i+1}\right\}$ so that it has direction $L_{7}$ or $L_{1}$, respectively.

By Property 3, it can easily be seen that such an edge must exist. It remains to be shown that $s_{i+1}$ is in $W$. By the given rules and the definition of $W_{1}, W_{2}$ and $W_{3}$, the only way $s_{i+1}$ could be outside $W$ would be if $s_{i+1}=\left(s_{i+1_{x}}, s_{i+1_{y}}\right)$ with $\left|s_{i+1_{x}}\right| \leq 1$ and $s_{i+1_{y}}<\left|s_{i+1_{x}}\right|$. If $s_{i+1_{x}}>0$, this would imply that $s_{i} \in W_{1}$ and that $\left\{s_{i}, s_{i+1}\right\}$ has direction $L_{6}$. Consider the unit length line segment in the direction of $L_{6}$ from $c$ to $d$ where $c=(-1 / 2,1 / 2)$. Since this segment is parallel to $\left\{s_{i}, s_{i+1}\right\}$, is at least as long, and since $c$ is below and to the right of every point in $W_{1}, d$ must be below and to the right of $s_{i+1}$. Hence the coordinates of $d=\left(d_{x}, d_{y}\right)$ must also satisfy $d_{y}<d_{x}$. But in contradiction we have $d_{x}=1 / 2$ and $d_{y}=1 / 2$. So it holds $s_{i+1} \in W$. The same holds if $s_{i+1_{x}}<0$.

Therefore, $\left\{s_{i}, s_{i+1}\right\}$ is a new edge of $T_{o p t}$ in $W$. We continue adding new edges to the path until a terminal is reached. Since we have at most $|K|-2$ Steiner points, the path must terminate with a terminal.

Let $\alpha \geq 0$ be arbitrary. We define $W(\alpha)$ by $W(\alpha)=\{(x, y) \in W \mid y \leq \alpha\}$. By the preceding arguments, we have the following result.

Corollary 1. Let $T_{\text {opt }}$ be a Steiner minimum tree for $K$ with $m\left(T_{o p t}\right) \leq 1$ and suppose $s_{0}=(0,0)$ is a Steiner point of $T_{\text {opt }}$. Then for any $\alpha \geq 0$ either $W(\alpha+1)$ contains a terminal of $K$ or $W(\alpha+1)-W(\alpha)$ contains a Steiner point of $T_{\text {opt }}$.

If $s$ is a Steiner point of a Steiner minimum tree $T_{o p t}$ for $K$ and $\{s, t\}$ is an edge of $T_{\text {opt }}$, let $O(s, t)$ denote the closed regular octagonal region of side length 2 which is bisected by the line through $s$ and $t$ and which intersects $\{s, t\}$ in the single point $s$. See Fig. 14.

Lemma 5. Let $T_{\text {opt }}$ be a Steiner minimum tree for $K$ with $m\left(T_{\text {opt }}\right) \leq 1$. If $s$ is a Steiner point of $T_{\text {opt }}$ and $\{s, t\}$ is an edge of $T_{\text {opt }}$, then $O(s, t)$ contains a terminal of $T_{\text {opt }}$. 


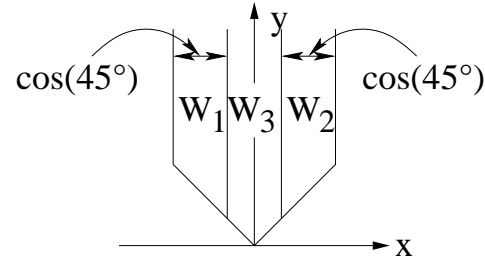

Fig. 13. Partitioning the strip $W$.

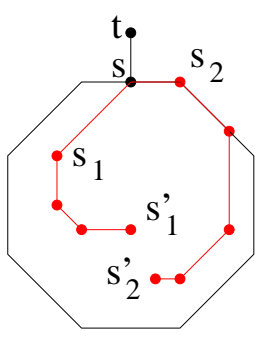

Fig. 14. The region $O(s, t)$

Proof. Suppose $O(s, t)$ contains no terminal of $T_{\text {opt }}$. Then $T_{\text {opt }}$ contains two edges $\left\{s, s_{1}\right\}$ and $\left\{s, s_{2}\right\}$ which lie inside or on the boundary of $O(s, t)$, since no edge is longer than 1. Without loss of generality $s_{1}$ lies to the left of $s$ and $s_{2}$ to the right. Since $O(s, t)$ contains no terminal, $s_{1}$ and $s_{2}$ must be Steiner points. We choose two paths $P_{1}$ and $P_{2}$ beginning at $s_{1}$ and $s_{2}$, respectively. For the path $P_{1}$ we choose the edges with smallest angles between the two edges on the right of them. For the path $P_{2}$ we choose edges with smallest angles between the two edges on the left of them. The paths $P_{1}$ and $P_{2}$ respectively ends if the last chosen edge is horizontal or has slope 1 . Since every edge is not longer than 1 the so chosen paths lay completely within $O(s, t)$. See Fig. 14. Let $s_{1}^{\prime}$ and $s_{2}^{\prime}$ be the two other endpoints of the paths $P_{1}$ and $P_{2}$.

Without loss of generality let $s_{1_{y}}^{\prime} \geq s_{2_{y}}^{\prime}$. We apply Lemma 4 to the point $s_{1}^{\prime}$. We can place the strip $W$ so that the point $s_{1}^{\prime}$ plays the role of $s_{0}=(0,0)$. The strip can be rotated by angles of $45^{\circ}$ so that the last edge except the point $s_{1}^{\prime}$ of $P_{1}$ is not inside the strip. By Lemma 4, there must exist a terminal $t^{\prime} \in K$ such that the path $P_{T_{o p t}}\left(s_{1}^{\prime}, t^{\prime}\right)$ lies entirely in $W$. Since the last edge of $P_{1}$ is not in $W$, no edge of $P_{T_{o p t}}\left(s_{1}^{\prime}, t^{\prime}\right)$ can intersect $P_{1} \cup\left\{s, s_{1}\right\}$ or $P_{2} \cup\left\{s, s_{2}\right\}$, since otherwise $T_{\text {opt }}$ would contain a cycle. Thus $P_{T_{\text {opt }}}\left(s_{1}^{\prime}, t^{\prime}\right) \subseteq O(s, t)$ which implies $t^{\prime} \in O(s, t)$. This contradicts the assumption that no terminal lies in $O(s, t)$.

For a point $x$ in the plane, let $D_{x}$ denote the disc of all points at a distance of at most 3. Then we have the following Corollary of Lemma 5.

Corollary 2. Let $s$ be a Steiner point of a Steiner minimal tree $T_{\text {opt }}$ for $K$ with $m\left(T_{\text {opt }}\right) \leq 1$. Then $D_{s}$ contains a terminal of $K$.

A probe $P$ consists of a copy of $W(10)$ together with the set of all points at a distance of at most 3 from some point of $W(10)-W(9)$. The point $p$ at the angle $90^{\circ}$ of the probe is called the tip of the probe. See Fig. 2.

Lemma 2. If $p$ is a Steiner point of an octilinear Steiner minimum tree with $m\left(T_{\text {opt }}\right) \leq$ 1 , then there must be at least one terminal located inside every valid probe $P$ with tip $p$.

Proof. Suppose $p$ is a Steiner point of $T_{\text {opt }}$ and $P$ contains no terminal. Since the strip $W(10)$ contains no terminal, by Corollary $1, W(10)-W(9)$ must contain a Steiner point $s$ of $T_{o p t}$. By Corollary 2, some terminal $t$ must be within distance 3 from $s$, so that $t \in P$. This contradicts the assumption that no terminal is contained in the probe $P$. 


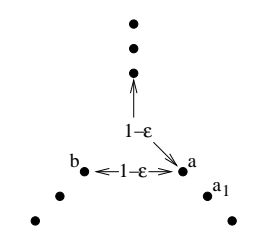

Fig. 15. Edge $\left\{a_{1}, b\right\}$ is longer than 1 .

\subsection{Properties of $T_{\text {opt }}(K(\mathcal{F}))$}

Let $\mathcal{F}=\left\{F_{1}, F_{2}, \ldots, F_{t}\right\}$ be a family of 3-sets $F_{k} \subseteq\{1,2, \ldots, 3 n\}$ and $T_{\text {opt }}$ a minimum 4-Steiner tree for $K(\mathcal{F})$. In this section we prove properties of $T_{\text {opt }}$.

By the construction of the set of terminals $K$ it is easy to see, that the longest edge in a minimum spanning tree for $K$ has length 1 . Hence by Lemma 3 ,

$$
m\left(T_{o p t}\right) \leq 1 .
$$

Thus, Lemma 2 can be applied to delimit the possible locations of Steiner points in $T_{\text {opt }}$. For almost every point $p$ of the plane not belonging to $K(\mathcal{F})$, the probe can be placed with $p$ at the probe tip and no point of $K$ which lies inside the probe. It follows that there are just two types of possibilities for a Steiner point $s$ of $T_{o p t}$ :

(i) $s$ is the Steiner point in the shortest connection of the three active points of a triangle $R$ or $R(\epsilon)$.

(ii) $s$ is the Steiner point along the shortest connection of the four active points of a square $Q(\epsilon)$.

Lemma 6. If $s, t \in K$ and the distance between $s$ and $t$ does not exceed $1 / 10$, then $\{s, t\}$ is an edge of $T_{\text {opt }}$.

Proof. Obviously, there is a spanning tree containing the edge $\{s, t\}$. By Lemma 3 , it follows $m\left(P_{T_{o p t}}(s, t)\right) \leq 1 / 10$. Suppose $\{s, t\}$ is not contained in $T_{o p t}$. The terminals $s$ and $t$ are connected by a path not containing an edge longer than $1 / 10$ and not containing the edge $\{s, t\}$. By the construction of $K$ no such path can only contain edges of pairs of active terminals. Therefore $P_{T_{o p t}}(s, t)$ contains a Steiner point. Due to the very restricted possible positions of Steiner points, the length of at least one of the two edges of $P_{T_{o p t}}(s, t)$ incident to the Steiner point must be longer than $1 / 10$ which contradicts $m\left(P_{T_{o p t}}(s, t)\right) \leq 1 / 10$.

Lemma 7. Every edge of $T_{\text {opt }}$ longer than $1 / 10$ that connects two terminals of $K$ connects two active terminals of the same square or triangle.

Proof. The edges known to be in $T_{o p t}$ by Lemma 6 form disjoint subtrees of $T_{o p t}$ that contain all terminals of $K$. No two terminals of $K$ in the same subtree can be connected by an additional edge (longer than $1 / 10$ ) since that would form a cycle. By the construction of $K$, since $\epsilon<1 / 200$, the only pairs of terminals in different subtrees, which are separated with distance 1 or less, are active terminals of the same square or triangle. See also Figure 15. Since $m\left(T_{o p t}\right) \leq 1$, the Lemma follows. 
$\mathrm{R}(\varepsilon)$

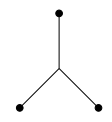

$\left(\alpha_{1}\right)$

$\mathrm{R}(0)$

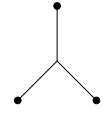

$\left(\beta_{1}\right)$

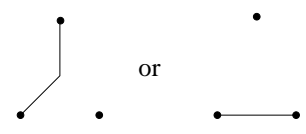

$\left(\alpha_{2}\right)$

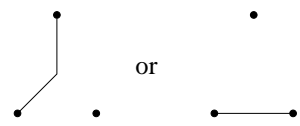

$\left(\beta_{2}\right)$

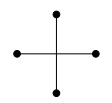

$\left(\gamma_{1}\right)$

$\mathrm{Q}(\varepsilon)$

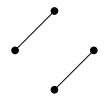

$\left(\gamma_{3}\right)$

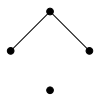

$\left(\gamma_{2}\right)$

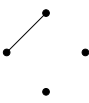

$\left(\gamma_{4}\right)$

Fig. 16. Possible configurations in active regions.

Lemma 8. The only terminals in $K$ adjacent to a Steiner point are the active terminals of the squares and triangles.

Proof. Since $m\left(T_{\text {opt }}\right) \leq 1$, any point $t$ adjacent to a Steiner point $s$ must be within distance 1 of one of the locations where a Steiner point can occur. If $t$ is not an active point, then there must be a point $t^{\prime} \in K$ in the same standard row at distance 1/10 from $t$ and closer to the corresponding active point than $t$. By Lemma $6, T_{\text {opt }}$ contains the edge $\left\{t, t^{\prime}\right\}$. But then, in either case, $T_{\text {opt }}$ contains two edges $\left\{t, t^{\prime}\right\}$ and $\{t, s\}$, that meet at a common point $t$ at an angle less than $90^{\circ}$. But then $T_{\text {opt }}$ is not minimum. So $t$ must be an active point.

Therefore, the Steiner tree $T_{\text {opt }}$ consists of all edges connecting two terminals of $K$ which have distance $1 / 10$ or less to each other and of edges connecting active terminals of the same square or triangle. We catalog the possible topologies of edges joining active terminals and (possibly) Steiner points within each type of active region in Fig. 16. Only one representative is given for symmetric configurations. Since every edge in $T_{\text {opt }}$ has length 1 or less, only configurations of edges with this property are shown. In Table 1, we list the total length of the edges in each configuration shown in Fig. 16.

\begin{tabular}{clrl} 
Configuration & \multicolumn{1}{c}{ Total Length } \\
\hline$\alpha_{1}$ & $l\left(\alpha_{1}\right)=\frac{\sqrt{2}+2}{\sqrt{2}}(1-\epsilon)$ \\
$\beta_{1}$ & $l\left(\beta_{1}\right)=\frac{\sqrt{2}+2}{2}$ \\
$\gamma_{1}$ & $l\left(\gamma_{1}\right)=2 \sqrt{2}(1-\epsilon)$ \\
$\alpha_{2}$ & $l\left(\alpha_{2}\right)=(1-\epsilon)$ \\
$\beta_{2}$ & $l\left(\beta_{2}\right)=1$ \\
$\gamma_{2}$ & $l\left(\gamma_{2}\right)=2(1-\epsilon)$ \\
$\gamma_{3}$ & $l\left(\gamma_{3}\right)=2(1-\epsilon)$ \\
$\gamma_{4}$ & $l\left(\gamma_{4}\right)=1-\epsilon$
\end{tabular}

Table 1. Lengths of configurations in Fig. 16. 


\subsection{The value of $l\left(T_{\text {opt }}\right)$}

Before estimates on the length of $T_{\text {opt }}$ can be given, we must specify a value for $\epsilon$. Let $\hat{C}=\hat{C}(K)$ the number of crossovers in $K=K(\mathcal{F})$. As noted above, $\hat{C} \leq 9 n t$. We choose $\epsilon$ as follows:

$$
\epsilon=\frac{1}{200 n t} \text {. }
$$

The choice of $\epsilon$ satisfies the inequality $\epsilon<1 / 200$ required by the construction of $R(\epsilon)$ and $Q(\epsilon)$.

Lemma 9. For $\epsilon=1 /(200 n t)$ we have

$$
\left(l\left(\beta_{1}\right)-l\left(\alpha_{1}\right)\right) \hat{U} \leq 2 / 3 l\left(\gamma_{1}\right)-l\left(\alpha_{1}\right) .
$$

Proof. We have $\left(l\left(\beta_{1}\right)-l\left(\alpha_{1}\right)\right) \hat{U}+l\left(\alpha_{1}\right) \leq\left(1+\frac{1}{\sqrt{2}}\right) 9 n t \epsilon+\left(1+\frac{1}{\sqrt{2}}\right)(1-\epsilon)$ and $l\left(\gamma_{1}\right)=$ $2 \sqrt{2}(1-\epsilon)$. To prove the Lemma, it is therefore sufficient to show that $\left(1+\frac{1}{\sqrt{2}}\right) 9 n t \epsilon+$ $\left(1+\frac{1}{\sqrt{2}}\right)(1-\epsilon) \leq \frac{4 \sqrt{2}}{3}(1-\epsilon)$ holds. The choice of $\epsilon$ implies this inequality.

By Lemma $6, T_{o p t}$ contains all edges which connect adjacent terminals of the same standard or long row. Let $L_{0}$ be the length of all such edges and $G$ the graph composed of all these edges. By the construction of $K$, a connected component of $G$ lies to the left of each level. Since $3 n+1$ levels exist, these are $3 n+1$ connected components. The connected component containing the upward terminators $\Omega_{0}, \Omega_{1}, \ldots, \Omega_{t}$ which is connected to the downward terminator $\Omega_{0}^{\prime}$, is contained in one of the $3 n+1$ connected components. Furthermore, for every crossover there exists a connected component below the crossover and one connected component to the right of the crossover. Therefore, we have $N=2 \hat{C}+3 n+1$ connected components in $G$.

All $N$ connected components must be joined in $T_{\text {opt }}$. This is done by connections between active terminals, shown in Fig. 16. Let $L_{1}$ denote the total length of all these connections, so that $l\left(T_{\text {opt }}\right)=L_{0}+L_{1}$. The following theorem finishes our proof that the octilinear Steiner tree decision problem is NP-complete.

Theorem 2. If $\mathcal{F}$ has an exact cover, then:

$$
l\left(T_{\text {opt }}\right) \leq 3 n l\left(\alpha_{1}\right)+(\hat{C}-3 n) l\left(\beta_{1}\right)+n l\left(\gamma_{1}\right)+L_{0} .
$$

If $\mathcal{F}$ does not contain an exact cover, then:

$$
l\left(T_{\text {opt }}\right) \geq 3 n l\left(\alpha_{1}\right)+(\hat{C}-3 n) l\left(\beta_{1}\right)+n l\left(\gamma_{1}\right)+L_{0}+\epsilon .
$$

Proof. Suppose there exists an exact cover $\mathcal{F}^{\prime}=\left\{F_{i_{1}}, F_{i_{2}}, \ldots, F_{i_{n}}\right\}$ for $\mathcal{F}$. We construct a Steiner tree $T$ for $K$ satisfying the inequality (3). Therefore, (3) holds also for $T_{o p t}$.

First we add all edges to $T$ which connect adjacent terminals of the same standard or long row. These edges have the total length $L_{0}$. The remaining edges are constructed as follows:

(i) In each square $Q_{i_{k}}, 1 \leq k \leq n$, form a $\gamma_{1}$-configuration.

(ii) In each crossover, that belongs to one of the three vertical chains of crossovers

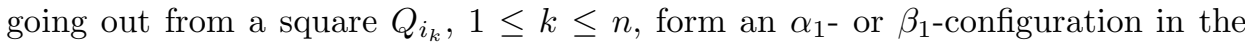
upper triangle of every crossover. 
(iii) In each crossover, not considered in (ii), form a $\beta_{1}$-configuration in the lower triangle.

Since each vertical chain of crossovers contains exactly one small crossover and $\mathcal{F}^{\prime}$ is an exact cover of $\mathcal{F}$, by (ii) and (iii) exactly $3 \mathrm{n} \alpha_{1}$-configurations are formed. The total length of the edges in $T$ is $3 n l\left(\alpha_{1}\right)+(\hat{C}-3 n) l\left(\beta_{1}\right)+n l\left(\gamma_{1}\right)+L_{0}$, as desired.

The component composed of the terminators $\Omega_{0}^{\prime}, \Omega_{0}, \Omega_{1}, \ldots, \Omega_{t}$ and the long rows connecting these, is called base. To see that we have constructed a Steiner tree for $K$, it is sufficient to show, that every of the $N$ components is connected to the base by a path.

Since each crossover contains either an $\alpha_{1^{-}}$or $\beta_{1}$-configuration in one of its triangles, all components at the $i$ th level, $0 \leq i \leq 3 n$, are joined together in an $i$ th level component. The 0th level component is connected with the base by the downward terminator $\Omega_{0}^{\prime}$. If $F_{k}=\left\{a_{k}, b_{k}, c_{k}\right\} \in \mathcal{F}^{\prime}$, then by (ii) the downward terminators $\bar{\Omega}_{k}, \bar{\Omega}_{k}^{\prime}$ and $\bar{\Omega}_{k}^{\prime \prime}$ are connected with the crossovers $U_{k}\left(a_{k}-1\right), U_{k}^{\prime}\left(b_{k}-1\right)$ and $U_{k}^{\prime \prime}\left(c_{k}-1\right)$ by $\alpha_{1}$-configurations in the upper triangles of the crossovers. Therefore, the $a_{k}$ th level components are connected with the $\left(a_{k}-1\right)$ th level components, the $b_{k}$ th level components with the $\left(b_{k}-1\right)$ th level components and the $c_{k}$ th level components with the $\left(c_{k}-1\right)$ th level components. Since each integer $i, 1 \leq i \leq 3 n$, belongs to some $F_{k} \in \mathcal{F}^{\prime}$, it follows by induction that all level components are connected with the base. We denote the connected component containing the base and all level components as skeleton. The only components remaining to be accounted for are those lying between successive levels. These are the long rows between the crossovers $U_{k}(i)$ and $U_{k}(i+1)$ and those between the squares $Q_{k}$ and the 0th level. In every vertical chain of crossovers, all crossovers contain an $\alpha_{1}$ - or $\beta_{1}$-configuration either exclusively in the upper triangles or exclusively in the lower triangles. By this it follows that each long row between the crossovers $U_{k}(i)$ and $U_{k}(i+1)$ is connected with an crossover. If $F_{k}=\left\{a_{k}, b_{k}, c_{k}\right\} \in \mathcal{F}^{\prime}$ then by (i) all rows adjacent to $Q_{k}$ are contained with $Q_{k}$ in the same connected component. Therefore $Q_{k}$ is in the same connected component as the base. If $F_{k}=\left\{a_{k}, b_{k}, c_{k}\right\} \notin \mathcal{F}^{\prime}$ then by (iii) the long rows on the left, on the right and above $Q_{k}$ are connected with the 0 th level. The long row below $Q_{k}$ is connected with the base. Since $\mathcal{F}^{\prime}$ is an exact cover, for each $i=1, \ldots, n$ exactly one downward terminator $\bar{\Omega}_{k}, \bar{\Omega}_{k}^{\prime}$ or $\bar{\Omega}_{k}^{\prime \prime}, 1 \leq k \leq t$, is connected by a long row with the preceding level. Therefore the terminators does not form a cycle. By the construction it follows that the remaining graph also does not contain a cycle and therefore is a Steiner tree for $K$.

Thus we have shown that (3) holds if $\mathcal{F}$ contains an exact cover. We shall now show that $\mathcal{F}$ contains an exact cover if (4) does not hold. If (4) does not hold, we must have:

$$
L_{1}<3 n l\left(\alpha_{1}\right)+(\hat{C}-3 n) l\left(\beta_{1}\right)+n l\left(\gamma_{1}\right)+\epsilon .
$$

For each configuration in Figure 16, let $N(\omega)$ the number of active regions containing a configuration $\omega$. Then we have:

$$
L_{1}=\sum_{w} N(w) l(w) .
$$

If we consider only the part of $T_{\text {opt }}$ containing edges of length $1 / 10$ or less, then we have, as noted earlier, $N=2 \hat{C}+3 n+1$ connected components. These $N$ components are connected in $T_{o p t}$ by configurations from Figure 16 which are contained in active 
regions. Considering the number of connected components joined by each configuration, we have:

$$
\begin{aligned}
N-1= & 2 N\left(\alpha_{1}\right)+2 N\left(\beta_{1}\right)+3 N\left(\gamma_{1}\right)+N\left(\alpha_{2}\right) \\
& +N\left(\beta_{2}\right)+2 N\left(\gamma_{2}\right)+2 N\left(\gamma_{3}\right)+N\left(\gamma_{4}\right) .
\end{aligned}
$$

The integer multiplier in (7) for each configuration is the reduction of the number of connected components obtained by a configuration. The multiplier is therefore one less than the number of connected components joined by this configuration. The following chain of inequalities holds for the lengths of the different types of configurations:

$$
\frac{1}{2} l\left(\alpha_{1}\right)<\frac{1}{2} l\left(\beta_{1}\right)<\frac{1}{3} l\left(\gamma_{1}\right)<\frac{1}{2} l\left(\gamma_{2}\right)=l\left(\alpha_{2}\right)=l\left(\gamma_{4}\right)=\frac{1}{2} l\left(\gamma_{3}\right)<l\left(\beta_{2}\right) .
$$

There are $\hat{C}$ crossovers in $K$ and thus $2 \hat{C}$ triangles. Each crossover contains at most one $\alpha_{1}$ - or $\beta_{1}$-configuration, since otherwise $T_{o p t}$ contains a cycle. Thus there can be at most $\hat{C}$ many $\alpha_{1}$ - and $\beta_{1}$-configurations. Therefore, we obtain

$$
N\left(\alpha_{1}\right)+N\left(\beta_{1}\right) \leq \hat{C} .
$$

Claim: we must have equality in (9), to hold (5). Thus suppose $N\left(\alpha_{1}\right)+N\left(\beta_{1}\right) \leq$ $\hat{C}-1$. Then we have by $(7)$ and the equality $N=2 \hat{C}+3 n+1$ :

$$
\begin{aligned}
2 \hat{C}+3 n-2 N\left(\alpha_{1}\right)-2 N\left(\beta_{1}\right)= & 3 N\left(\gamma_{1}\right)+N\left(\alpha_{2}\right)+N\left(\beta_{2}\right) \\
& +2 N\left(\gamma_{2}\right)+2 N\left(\gamma_{3}\right)+N\left(\gamma_{4}\right) .
\end{aligned}
$$

Together with (6) and (8) we obtain

$$
\begin{array}{ll}
= & N\left(\alpha_{1}\right) l\left(\alpha_{1}\right)+N\left(\beta_{1}\right) l\left(\beta_{1}\right)+N\left(\gamma_{1}\right) l\left(\gamma_{1}\right)+N\left(\alpha_{2}\right) l\left(\alpha_{2}\right) \\
& +N\left(\beta_{2}\right) l\left(\beta_{2}\right)+N\left(\gamma_{2}\right) l\left(\gamma_{2}\right)+N\left(\gamma_{3}\right) l\left(\gamma_{3}\right)+N\left(\gamma_{4}\right) l\left(\gamma_{4}\right) \\
=\quad & N\left(\alpha_{1}\right) l\left(\alpha_{1}\right)+N\left(\beta_{1}\right) l\left(\beta_{1}\right)+\frac{3}{3} N\left(\gamma_{1}\right) l\left(\gamma_{1}\right)+N\left(\alpha_{2}\right) l\left(\alpha_{2}\right) \\
& +N\left(\beta_{2}\right) l\left(\beta_{2}\right)+\frac{2}{2} N\left(\gamma_{2}\right) l\left(\gamma_{2}\right)+\frac{2}{2} N\left(\gamma_{3}\right) l\left(\gamma_{3}\right)+N\left(\gamma_{4}\right) l\left(\gamma_{4}\right) \\
& N\left(\alpha_{1}\right) l\left(\alpha_{1}\right)+N\left(\beta_{1}\right) l\left(\beta_{1}\right)+\left(3 N\left(\gamma_{1}\right)+N\left(\alpha_{2}\right)\right. \\
& \left.+N\left(\beta_{2}\right)+2 N\left(\gamma_{2}\right)+2 N\left(\gamma_{3}\right)+N\left(\gamma_{4}\right)\right) \frac{1}{3} l\left(\gamma_{1}\right) \\
& N\left(\alpha_{1}\right) l\left(\alpha_{1}\right)+N\left(\beta_{1}\right) l\left(\beta_{1}\right)+\left(2 \hat{C}-2-2 N\left(\alpha_{1}\right)\right. \\
& \left.-2 N\left(\beta_{1}\right)\right) \frac{1}{3} l\left(\gamma_{1}\right)+\frac{3 n+2}{3} l\left(\gamma_{1}\right)
\end{array}
$$

$$
N\left(\alpha_{1}\right)+\underset{>}{N\left(\beta_{1}\right) \leq \hat{C}-1}(\hat{C}-1) l\left(\alpha_{1}\right)+\frac{3 n+2}{3} l\left(\gamma_{1}\right) .
$$


However, by Lemma 9 the choice of $\epsilon$ insures $\left(l\left(\beta_{1}\right)-l\left(\alpha_{1}\right)\right) \hat{C}<\frac{2}{3} l\left(\gamma_{1}\right)-l\left(\alpha_{1}\right)$. This implies:

$$
\begin{aligned}
& L_{1} \quad>\quad(\hat{C}-1) l\left(\alpha_{1}\right)+\frac{3 n+2}{3} l\left(\gamma_{1}\right) \\
& >\quad \hat{C} l\left(\beta_{1}\right)+n l\left(\gamma_{1}\right) \\
& =\quad 3 n l\left(\beta_{1}\right)+(\hat{C}-3 n) l\left(\beta_{1}\right)+n l\left(\gamma_{1}\right) \\
& l\left(\alpha_{1}\right)=l\left(\beta_{1}\right)-\frac{\sqrt{2}+2}{2} \epsilon \ln l\left(\alpha_{1}\right)+(\hat{C}-3 n) l\left(\beta_{1}\right)+n l\left(\gamma_{1}\right)+3 n \frac{\sqrt{2}+2}{2} \epsilon .
\end{aligned}
$$

This contradicts (5). Thus if (5) holds, then we have:

$$
N\left(\alpha_{1}\right)+N\left(\beta_{1}\right)=\hat{C} .
$$

Because of (11), each crossover in $T_{o p t}$ contains exactly one $\alpha_{1}$ - or $\beta_{1}$-configuration. Therefore, all components at the same level are connected to each other. In every level there exists at most one $\alpha_{1}$-configuration, since two of this configurations at the same level would form a cycle with the edges at the next level. $\left(\alpha_{1}\right.$-configurations can exist only in small upper triangles of crossovers. These small triangles are connected with a downward terminator at the next level above. Two of these connections would form a cycle.) Therefore, it holds $N\left(\alpha_{1}\right) \leq 3 n$, since there exist $3 n+1$ levels and the $3 n$th level does not contain crossovers. In a similar way as before it can be shown, that equality holds. Since suppose $N\left(\alpha_{1}\right) \leq 3 n-1$. Then it holds by (6), (8), (10) and (11):

$$
\begin{aligned}
& L_{1} \quad>\quad(3 n-1) l\left(\alpha_{1}\right)+(\hat{C}-3 n+1) l\left(\beta_{1}\right)+n l\left(\gamma_{1}\right)
\end{aligned}
$$

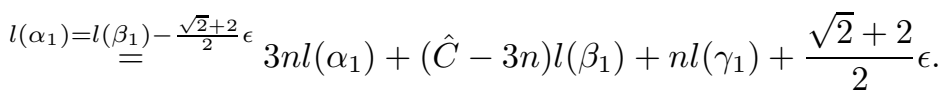

This contradicts (5). Thus if (5) holds, then we have:

$$
N\left(\alpha_{1}\right)=3 n
$$

By this equality and by $(5),(8)$ and $\frac{1}{2} l\left(\gamma_{2}\right)>\frac{1}{3} l\left(\gamma_{1}\right)+\epsilon$, it follows:

$$
\begin{gathered}
N\left(\beta_{1}\right)=\hat{C}-3 n, \quad N\left(\gamma_{1}\right)=n, \\
N\left(\alpha_{2}\right)=N\left(\beta_{2}\right)=N\left(\gamma_{2}\right)=N\left(\gamma_{3}\right)=N\left(\gamma_{4}\right)=0 .
\end{gathered}
$$

If $C_{k}(i), i>0$, is a crossover, which contains the Steiner point in the upper triangle, then $C_{k}(i-1)$ must contain the Steiner point in the upper triangle as well, since otherwise the horizontal long row between the two crossovers is not joined to the rest of the tree, since by $(13) N\left(\beta_{2}\right)=0$ and $N\left(\alpha_{2}\right)=0$. By induction it follows that the active terminals of the square $Q_{k}$ are joined together. Thus $Q_{k}$ contains a $\gamma_{1}$-configuration, since by (13) $N\left(\gamma_{2}\right)=N\left(\gamma_{3}\right)=N\left(\gamma_{4}\right)=0$. By (12) for each $k, 1 \leq k \leq 3 n$, the $(k-1)$ th level contains an $\alpha_{1}$-configuration, i.e., a small crossover with a Steiner point in the upper small triangle. As noted earlier, this means, that the square $Q_{i}$ below the crossover contains a $\gamma_{1}$-configuration. By the construction of $K, k$ must be an element of the 3 -set $F_{i_{j}}$ corresponding to the square $Q_{i_{j}} . n$ squares $Q_{i_{j}}$ contain joined active terminals. The sets corresponding to these squares must be disjoint, since otherwise $T_{\text {opt }}$ must contain a cycle. Thus the sets $F_{i_{j}}$ cover $\{1,2, \ldots, 3 n\}$. Thus $(5)$ implies that an exact cover of $\mathcal{F}$ exists. 

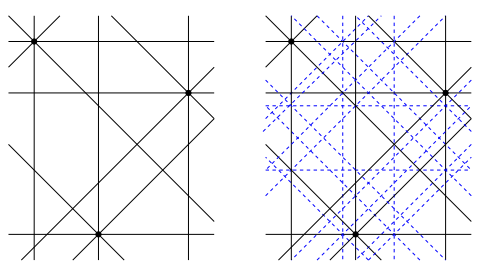

Fig. 17. Example of the graph $G_{1}$ for a set of three terminals (left) and its refinement $G_{1}^{k}$ with $k=2$ (right).

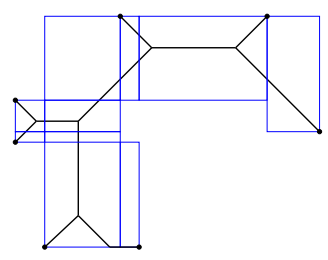

Fig. 18. Example of the covering of an octilinear Steiner tree by rectangles with edges in $G_{1}^{k}$.

\section{Error Bounds for Graph-Based Approximations}

In this section we show how to improve upon the approximation guarantee obtained for $G_{1}(K)$ for octilinear Steiner trees. To this end we construct a graph $G_{1}^{k}(K)$ which is parameterized by some constant $k$.

Recall from the Introduction that the graph $G_{1}$ is the graph induced by four lines (vertical, horizontal, and both main diagonals) through each terminal. The idea is to refine $G_{1}$ by superimposing $O(k)$ additional lines. This is done as follows. Given a set of terminals $K$ with $|K|=n$, let $B B(K)$ denote the bounding box of this point set, that is, the smallest axis-parallel rectangle which includes all terminals. We subdivide each side of $B B(K)$ equidistantly with $k$ points into $k+1$ segments and add for each subdivision point additional lines in all four feasible orientations of the octilinear geometry. See Fig. 17 for a small example. Since we have $O(n+k)$ lines in each feasible direction, we get $O\left((n+k)^{2}\right)$ intersection points of these lines. Hence, the induced graph $G_{1}^{k}$ has $O\left((n+k)^{2}\right)$ many vertices and edges. For the bounding box $B B(K)$ with side lengths $b b_{x}$ and $b b_{y}$, denote by $b b:=\max \left\{b b_{x}, b b_{y}\right\}$ its maximum side length.

We next define how to cover a Steiner tree $T$ by a set of axis-parallel rectangles as follows (the rectangles may overlap). For each Steiner point $s$ of $T$, the set $\mathcal{R}$ contains a smallest rectangle including $s$ with horizontal and vertical edges from $G_{1}^{k}$. In the degenerate case that $s$ lies on a vertex or an edge of $G_{1}^{k}$ we add no rectangle. We also add a smallest enclosing rectangle for each point $p$ where an edge of $T$ bends. Degenerate cases are handled as with Steiner points. For each straight-line segment of $T$ not covered by previous rectangles we independently add to $\mathcal{R}$ a smallest enclosing rectangle bounded by vertical and horizontal edges from $G_{1}^{k}$. Thus, we finally have the following partition of the Steiner tree: $T=\cup_{R \in \mathcal{R}}(T \cap R)$. See Fig. 18 for an example.

For a given tree $T$, we construct an approximating Steiner tree $T_{a p p}$ with edges in $G_{1}^{k}$ as follows. For each rectangle $R \in \mathcal{R}$ let $S_{R}$ be the set of intersection points of $T_{\text {opt }}$ with the boundary of $R$. We connect the point set $S_{R}$ in the shortest possible way by (portions of) edges in $G_{1}^{k}$, yielding a tree $T_{R}$. From the union of all these trees $T_{R}$ we eliminate in a postprocessing step the longest edge of each cycle which may occur and all leaves and incident edges of the resulting tree which are not terminals. We thereby obtain our approximation $T_{a p p}$. The following technical lemma shows that we can bound for each rectangle $R$ included in $\mathcal{R}$ the length $\ell\left(T_{a p p} \cap R\right)$ of $T_{a p p} \cap R$ in terms of the length $\ell(T \cap R)$ of $T \cap R$. 


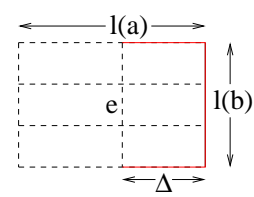

(a)

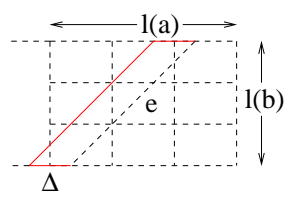

(b)

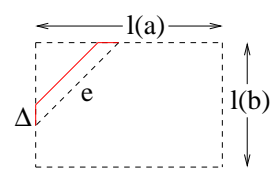

(c)

Fig. 19. Case 1 (i),(ii): $T \cap R$ does not contain a Steiner point and consists of exactly one straight edge.

Lemma 10. For each $R \in \mathcal{R}$, the following bound holds:

$$
\ell\left(T_{a p p} \cap R\right)-\ell(T \cap R) \leq(4-\sqrt{2}) \frac{b b}{k+1} .
$$

Proof. We consider an arbitrary rectangle $R \in \mathcal{R}$.

Case 1: $T \cap R$ does not contain a Steiner point.

We must distinguish four subcases:

(i) $T \cap R$ consists exactly of one horizontal or vertical edge $e$.

Without loss of generality let $e$ be a vertical edge. The endpoints of the edge $e$ can be connected as in Fig. 19(a). We denote by $\Delta$ the smallest distance from $e$ to a vertical edge of $G_{1}^{k}$. We have $\Delta \leq b b /(2(k+1))$ by the choice of $R$ and the construction of $G_{1}^{k}$. Then it holds:

$$
l\left(T_{a p p} \cap R\right)-l(T \cap R) \leq l(e)+2 \Delta-l(e) \stackrel{\Delta \leq \frac{b b}{2(k+1)}}{\leq} \frac{b b}{k+1} .
$$

(ii) $T \cap R$ consists of exactly one diagonal edge $e$.

If $l(e)=\sqrt{2} l(b)$ then the endpoints of $e$ can be connected in $G_{1}^{k}$ as in Fig. 19(b). We denote by $\Delta$ the smallest distance from $e$ to a parallel edge of $G_{1}^{k}$. We have $\Delta \leq$ $b b /(2(k+1))$. Then it holds:

$$
l\left(T_{a p p} \cap R\right)-l(T \cap R) \leq l(e)+2 \Delta-l(e) \stackrel{\Delta \leq \frac{b b}{2(k+1)}}{\leq} \frac{b b}{k+1} .
$$

If $l(e)<\sqrt{2} l(b)$ then the endpoints of $e$ can be connected in $G_{1}^{k}$ as in Fig. 19(c). We denote by $\Delta$ the smallest distance from $e$ to a parallel edge of $G_{1}^{k}$ with the same length or less than $e$. We have $\Delta \leq b b /(k+1)$. Then it holds:

$$
l\left(T_{a p p} \cap R\right)-l(T \cap R) \leq l(e)+(2-\sqrt{2}) \Delta-l(e) \stackrel{\Delta \leq \frac{b b}{k+1}}{\leq}(2-\sqrt{2}) \frac{b b}{k+1}
$$

(iii) $T \cap R$ consists of exactly one bending edge $e$ shown in Fig. 20(a).

Suppose the connection of the endpoints of $e$ in $G_{1}^{k}$ along the pointed edges in Fig. 20(b) is not longer than the connection along the pointed edges in Fig. 20(c). This is equivalent to $\Delta \leq \Delta^{\prime}$. Since $R$ is chosen as small as possible, we have $l(c) \leq b b /(k+1)$ and 


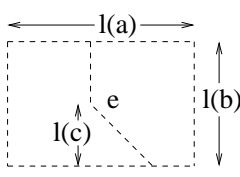

(a)

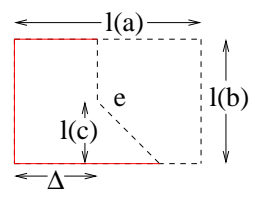

(b)

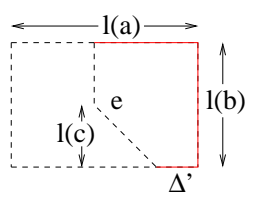

(c)

Fig. 20. Case 1 (iii): $T \cap R$ consists of exactly one bending edge between opposite sides of $R$.

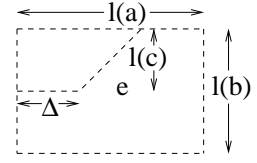

(a)

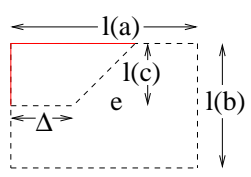

(b)

Fig. 21. Case 1 (iii): $T \cap R$ consists of exactly one bending edge between adjacent sides of $R$.

$\Delta \leq b b /(2(k+1))$. Then it holds:

$$
\begin{aligned}
l\left(T_{a p p} \cap R\right)-l(T \cap R) & \leq \quad l(b)+l(c)+2 \Delta-(l(b)+(\sqrt{2}-1) l(c)) \\
l(c) & \leq \frac{b b}{k+1} \\
\Delta \leq & \frac{b b}{2(k+1)} \\
& \leq
\end{aligned}
$$

Suppose the connection of the endpoints of $e$ in $G_{1}^{k}$ along the pointed edges in Fig. 20(c) is shorter as the connection along the pointed edges in Fig. 20(b). This is equivalent to $\Delta^{\prime}<\Delta$. Since $R$ is chosen as small as possible, we have $l(c) \leq b b /(k+1)$ and $\Delta^{\prime} \leq b b /(2(k+1))$. Then it holds:

$$
\begin{gathered}
l\left(T_{a p p} \cap R\right)-l(T \cap R) \quad \leq \quad l(b)+l(c)+2 \Delta^{\prime}-(l(b)+(\sqrt{2}-1) l(c)) \\
l(c) \leq \frac{b b}{k+1} \\
\Delta^{\prime} \leq \frac{b b}{2(k+1)}(3-\sqrt{2}) \frac{b b}{k+1} .
\end{gathered}
$$

(iv) $T \cap R$ consists exactly of one bending edge shown in Fig. 21(a). The endpoints of $e$ can be connected in $G_{1}^{k}$ by the pointed edges in Fig. 21(b). Since $R$ is chosen as small as possible, we have $l(c) \leq b b /(k+1)$. Then it holds:

$$
l\left(T_{a p p} \cap R\right)-l(T \cap R) \leq 2 l(c)+\Delta-(\sqrt{2} l(c)+\Delta) \stackrel{l(c) \leq \frac{b b}{k+1}}{\leq}(2-\sqrt{2}) \frac{b b}{k+1} .
$$

Case 2: $T \cap R$ contains at least one Steiner point.

We consider different subcases, depending on the number of sides by which $T$ intersects the boundary of $R$.

(i) $T$ intersects the boundary of $R$ at two different sides $a$ and $b$ of $R$.

Suppose the sides $a$ and $b$ are adjacent. We denote by $l(c)$ the maximum distance of 


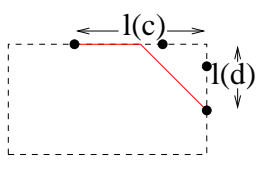

(a)

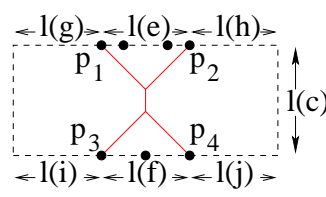

(b)

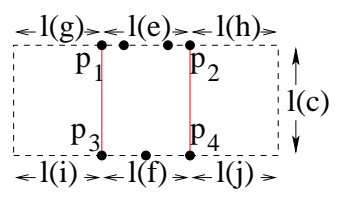

(c)

Fig. 22. Case 2: $T \cap R$ contains at least one Steiner point.

an intersection point of $T$ on side $a$ to the side $b$ and by $l(d)$ the maximum distance of an intersection point of $T$ on the side $b$ to the side $a$. Without loss of generality we let $l(c) \geq l(d)$. Then we have $l(T \cap R) \geq l(c)+(\sqrt{2}-1) l(d)$ and $l\left(T_{a p p} \cap R\right) \leq l(c)+l(d)$. See also Fig. 22(a). Then it holds:

$$
\begin{aligned}
l\left(T_{\text {app }} \cap R\right)-l(T \cap R) & \leq l(c)+l(d)-(l(c)+(\sqrt{2}-1) l(d)) \\
& =(2-\sqrt{2}) l(d) \\
& \leq(2-\sqrt{2}) \frac{b b}{k+1} .
\end{aligned}
$$

Suppose next that side $a$ lies opposite to $b$. Let $c$ and $d$ denote the remaining sides of $R$. We denote by $p_{1}$ and $p_{2}$ the two intersection points of $T$ with the side $a$ which have the maximum distance $l(e)$ between each other and by $p_{3}$ and $p_{4}$ we denote the two intersection points of $T$ with the side $b$ which have the maximum distance $l(f)$ between each other. We denote the other distances of the points to the adjacent edges as in Fig. $22(\mathrm{~b})$. Without loss of generality let $l(g)+l(i) \leq l(h)+l(j)$.

Suppose we have $l(e)+l(f) \leq 2 l(c)$. Then we have $l(T \cap R) \geq l(c)+(\sqrt{2}-1 / 2) l(e)+$ $(\sqrt{2}-1 / 2) l(f)$ and $l\left(T_{a p p} \cap R\right) \leq l(c)+l(e)+l(f)+l(g)+l(i)$. See Fig. $22(\mathrm{~b})$. Then it holds:

$$
\begin{aligned}
& l\left(T_{a p p} \cap R\right)-l(T \cap R) \quad \leq \quad l(c)+l(e)+l(f)+l(g)+l(i) \\
& -(l(c)+(\sqrt{2}-1 / 2) l(e) \\
& +(\sqrt{2}-1 / 2) l(f)) \\
& =\quad(3 / 2-\sqrt{2}) l(e)+(3 / 2-\sqrt{2}) l(f) \\
& +l(g)+l(i) \\
& \begin{array}{l}
l(e)+l(g) \leq \frac{b b}{k+1} \\
l(f)+l(i) \leq \frac{b b}{k+1}
\end{array} \\
& \begin{array}{c}
l(f)+l(i) \leq \frac{b b}{k+1} \\
\leq
\end{array}(3-2 \sqrt{2}) \frac{b b}{k+1}+(\sqrt{2}-1 / 2) l(g) \\
& +(\sqrt{2}-1 / 2) l(i) \leq 2 \frac{b b}{k+1} .
\end{aligned}
$$

Suppose we have $l(e)+l(f)>2 l(c)$. Then we have $l(T \cap R) \geq 2 l(c)$ and $l\left(T_{a p p} \cap R\right) \leq$ $l(c)+l(e)+l(f)+l(g)+l(i)$. See Fig. 22(c). Then it holds:

$$
\begin{aligned}
l\left(T_{a p p} \cap R\right)-l(T \cap R) & \leq l(c)+l(e)+l(f)+l(g)+l(i)-2 l(c) \\
& =l(e)+l(f)+l(g)+l(i)-l(c) \\
& <2 \frac{b b}{k+1} .
\end{aligned}
$$




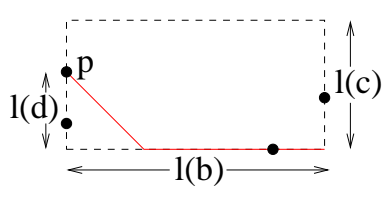

(a)

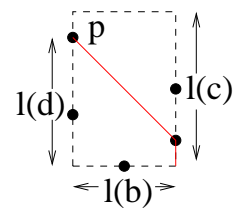

(b)

Fig. 23. Case 2 (ii): $T$ intersects the boundary of $R$ at three different sides.

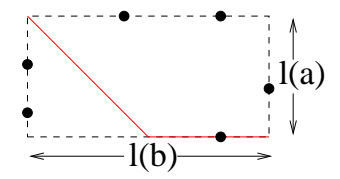

Fig. 24. Case 2 (iii): $T$ intersects the boundary of $R$ at all four sides.

(ii) $T$ intersects the boundary of $R$ at three different sides $a, b$ and $c$.

Without loss of generality, side $a$ lies opposite to $c$. Let $l(d)$ be the maximum distance of an intersection point $p$ of $T$ with side $a$ or $c$ to the side $b$. Without loss of generality $p$ lies on the side $a$.

Suppose it holds $l(a) \leq l(b)$. Then we have $l(T \cap R) \geq l(b)+(\sqrt{2}-1) l(d)$ and $l\left(T_{a p p} \cap\right.$ $R) \leq l(b)+2 l(d)$. See Fig. 23(a).

Then we obtain

$$
\begin{aligned}
l\left(T_{a p p} \cap R\right)-l(T \cap R) & \leq l(b)+2 l(d)-(l(b)+(\sqrt{2}-1) l(d)) \\
& =(3-\sqrt{2}) l(d) \\
& \leq(3-\sqrt{2}) \frac{b b}{k+1} .
\end{aligned}
$$

Suppose it holds $l(a)>l(b)$. Then we have $l(T \cap R) \geq(\sqrt{2}-1) l(b)+l(d)$ and $l\left(T_{\text {app }} \cap\right.$ $R) \leq l(b)+2 l(d)$. See Fig. 23(b). Then it holds:

$$
\begin{aligned}
l\left(T_{\text {app }} \cap R\right)-l(T \cap R) & \leq l(b)+2 l(d)-((\sqrt{2}-1) l(b)+l(d)) \\
& =(2-\sqrt{2}) l(b)+l(d) \\
& \leq(3-\sqrt{2}) \frac{b b}{k+1} .
\end{aligned}
$$

(iii) $T$ intersects the boundary of $R$ at all four sides. Let $l(a)$ and $l(b)$ the lengths of the sides of $R$. Without loss of generality $l(a) \leq l(b)$. Then we have $l(T \cap R) \geq$ $(\sqrt{2}-1) l(a)+l(b)$ and $l\left(T_{a p p} \cap R\right) \leq 2 l(a)+2 l(b)$. See Fig 24 . Then it holds:

$$
\begin{aligned}
l\left(T_{\text {app }} \cap R\right)-l(T \cap R) & \leq 2 l(a)+2 l(b)-((\sqrt{2}-1) l(a)+l(b)) \\
& =(3-\sqrt{2}) l(a)+l(b) \\
& \leq(4-\sqrt{2}) \frac{b b}{k+1} .
\end{aligned}
$$

This completes our case analysis. 
Lemma 11. The graph $G_{1}^{k}$ contains an octilinear Steiner tree which is at most a factor of

$$
1+\frac{(2 n-3)(4-\sqrt{2})}{k+1}
$$

longer than the optimal one.

Proof. Let $K$ be a set of points in the plane with $|K|=n$ and $T_{\text {opt }}$ be some octilinear Steiner minimum tree for $K$. We have to show that there is some octilinear Steiner tree $T_{a p p}$ within $G_{1}^{k}$ which approximates $T_{o p t}$ sufficiently well.

We cover $T_{\text {opt }}$ by a set $\mathcal{R}$ of axis-parallel rectangles as described above. Let us assume that $T_{\text {opt }}$ is composed of $k \geq 1$ full Steiner trees with $n_{1}, n_{2}, \ldots, n_{k} \geq 2$ vertices each. Then $\sum_{i=1}^{k} n_{i}=n+k-1$. Each full component may have at most $s_{i} \leq n_{i}-2$ Steiner points. Hence, the total number of Steiner points satisfies $\sum_{i=1}^{k} s_{i} \leq n-k-1$. If $m_{i}$ denotes the number of edges in the $i$-th full component, we have $m_{i}=n_{i}+s_{i}-1$ for a total of $m=\sum_{i=1}^{k} m_{i} \leq 2 n-2-k$ edges in $T_{\text {opt }}$.

The cover $\mathcal{R}$ of $T_{\text {opt }}$ by rectangles contains at most one rectangle per Steiner point, one rectangle for each edge and at most two additional rectangles per bending edge (one for the bending point and one for the second part of the edge). By Property 4, we may assume that each full component has at most one bending edge. Thus $|\mathcal{R}| \leq$ $\sum_{i=1}^{k} s_{i}+\sum_{i=1}^{k} m_{i}+2 k \leq 3 n-3$.

Next we analyze the length $\ell\left(T_{a p p}\right)$ of $T_{a p p}$ in comparison to the optimal length $\ell\left(T_{o p t}\right)$. All edges of $T_{\text {opt }}$ which are incident to a terminal are represented in $G_{1}^{k}$. Hence, for all corresponding rectangles $\ell\left(T_{\text {opt }} \cap \mathcal{R}\right)=\ell\left(T_{\text {app }} \cap \mathcal{R}\right)$. Clearly, there are at least $n$ edges incident to terminals. This implies, that for at most $2 n-3$ rectangles of $\mathcal{R}$ there will be a difference between $\ell\left(T_{a p p} \cap \mathcal{R}\right)$ and $\ell\left(T_{\text {opt }} \cap \mathcal{R}\right)$. Thus, we have

$$
\begin{aligned}
\frac{\ell\left(T_{a p p}\right)}{\ell\left(T_{o p t}\right)} & =\frac{\ell\left(T_{o p t}\right)+\sum_{R \in \mathcal{R}}\left(\ell\left(T_{a p p} \cap \mathcal{R}\right)-\ell\left(T_{o p t} \cap \mathcal{R}\right)\right)}{\ell\left(T_{o p t}\right)} \\
& \leq \frac{\ell\left(T_{o p t}\right)+(2 n-3) \cdot \max _{R \in \mathcal{R}}\left\{\ell\left(T_{a p p} \cap \mathcal{R}\right)-\ell\left(T_{o p t} \cap \mathcal{R}\right)\right\}}{\ell\left(T_{o p t}\right)} .
\end{aligned}
$$

Hence, it suffices to show that

$$
\max _{R \in \mathcal{R}}\left\{\ell\left(T_{a p p} \cap \mathcal{R}\right)-\ell\left(T_{o p t} \cap \mathcal{R}\right)\right\} \leq(4-\sqrt{2}) \cdot \frac{\ell\left(T_{o p t}\right)}{k+1} .
$$

This relation follows from Lemma 10 and the observation that $\ell\left(T_{o p t}\right) \geq b b$, since every Steiner tree must connect the terminals which define the bounding box $B B(K)$.

Theorem 3. For a given set of $n$ terminals in the plane and for every $\varepsilon>0$ there is a graph of size $O\left(\frac{n^{2}}{\varepsilon^{2}}\right)$ which contains a $(1+\varepsilon)$-approximation of a minimum octilinear Steiner tree.

Proof. The approximation guarantee follows directly from Lemma 11 if we choose $k:=$ $\frac{(4-\sqrt{2}) 2 n}{\varepsilon}$. With such a choice of $k$, the graph has the claimed size.

Blockages. Let us now sketch the necessary modifications in the presence of obstacles. Let $K$ be a set of points (terminals) in the plane and $O$ be a set of octilinear (or rectilinear) obstacles. Denote by $V_{O}$ the set of obstacle vertices. Let $n=|K|+\left|V_{O}\right|$. 
Analogously to the definition of $G_{1}(K)$, we now define a graph $G(K, O)$ which is induced by the set $L$ of lines in all feasible directions in 4-geometry going through terminals or obstacle vertices.

For a given parameter $k$, we refine $G(K, O)$ by adding lines. For any two parallel lines in $L$ which are neighbored (i.e., no third line with the same orientation lies between them) we add $k$ additional lines with the same orientation between them and place them equidistantly. In total, we have $O(n k)$ lines.

From the resulting induced graph, we erase all vertices and their incident edges which lie strictly inside some obstacle. The latter guarantees that every Steiner tree in this graph corresponds to a tree in the plane which avoids all obstacles.

Theorem 4. Let $\alpha$ denote the approximation guarantee for an algorithm solving the Steiner tree problem in graphs. Given a terminal set $K$, a set of octilinear obstacles $O$, and some $\varepsilon>0$, there is an $(\alpha+\varepsilon)$-approximation of the octilinear Steiner tree problem with obstacles which have to be avoided.

The proof of this theorem follows basically the same ideas as that for the case without obstacles. There is one essential difference, however. In the presence of obstacles, edges between terminals and/or Steiner points may be forced to bend several times. But if such an edge bends, then all but at most two of its straight segments will lie on $G(K, O)$. Since we have at most $2 n-3$ edges (each contributing two segments and possibly one corner point), but $n$ edges incident to terminals and $n-2$ Steiner points, a cover by rectangles of an octilinear Steiner minimum tree requires at most $3 \cdot(2 n-3)-n+n-2=6 n-11$ rectangles on which we have to find an approximative solution. This upper bound of $6 n-11$ rectangles (instead of $2 n-3$ without obstacles) suffices for an analogous result as in Lemma 11.

\section{Acknowledgment}

The first author was partially supported by the DFG Focus Program 1126 "Algorithmic Aspects of Large and Complex Networks", grant Mu1482/2-2.

\section{References}

[APD03] E. Althaus, T. Polzin, and S.V. Daneshmand. Improving linear programming approaches for the Steiner tree problem. Research Report MPI-I-2003-1-004, MaxPlanck-Institut für Informatik, Saarbrücken, Germany, March 2003.

[Aro98] S. Arora. Polynomial time approximation schemes for the Euclidean traveling salesman and other geometric problems. Journal of the ACM, 45:753-782, 1998.

[BTW00] M. Brazil, D.A. Thomas, and P. Winter. Minimum networks in uniform orientation metrics. SIAM Journal on Computing, 30(5):1579-1593, 2000.

[BTWZ02] M. Brazil, D.A. Thomas, J.F. Weng, and M. Zachariasen. Canonical forms and algorithms for Steiner trees in uniform orientation metrics. Technical Report TR02/22, DIKU, Department of Computer Science, Copenhagen, Denmark, 2002. To appear in Algorithmica.

[Cou03] C. Coulston. Constructing exact octagonal Steiner minimal trees. In ACM Great Lakes Symposium on VLSI, pages 1-6, 2003.

[DH92] D.-Z. Du and F.K. Hwang. Reducing the Steiner problem in a normed space. SIAM Journal on Computing, 21(6):1001-1007, 1992. 
[GGJ77] M.R. Garey, R.L. Graham, and D.S. Johnson. The complexity of computing Steiner minimal trees. SIAM Journal on Applied Mathematics, 32:835-859, 1977.

[GJ77] M.R. Garey and D.S. Johnson. The rectilinear Steiner tree problem is NP-complete. SIAM Journal on Applied Mathematics, 32:826-834, 1977.

[Han66] M. Hanan. On Steiner's problem with rectilinear distance. SIAM Journal on Applied Mathematics, 14:255-265, 1966.

[Kar72] R.M. Karp. Reducibility among combinatorial problems. In R.E. Miller and J.W. Thatcher, editors, Complexity of Computer Computations, pages 85-104. Plenum Press, New York, 1972.

[KMZ03] A.B. Kahng, I.I. Măndoiu, and A.Z. Zelikovsky. Highly scalable algorithms for rectilinear and octilinear Steiner trees. Proceedings 2003 Asia and South Pacific Design Automation Conference (ASP-DAC), pages 827-833, 2003.

[Koh95] C.K. Koh. Steiner problem in octilinear routing model. Master thesis, National University of Singapore, 1995.

[LS96] D.T. Lee and C.-F. Shen. The Steiner minimal tree problem in the $\lambda$-geometry plane. In Proceedings 7th International Symposium on Algorithms and Computations (ISAAC 1996), volume 1178 of Lecture Notes in Computer Science, pages 247-255. Springer, 1996.

[LX00] G.-H. Lin and G. Xue. Reducing the Steiner problem in four uniform orientations. Networks, 35(4):287-301, 2000.

[Mit99] J.S.B. Mitchell. Guillotine subdivisions approximate polygonal subdivisions: A simple polynomial-time approximation scheme for geometric TSP, $k$-MST, and related problems. SIAM Journal on Computing, 28(4):1298-1309, 1999.

[NWZ02] B.K. Nielsen, P. Winter, and M. Zachariasen. An exact algorithm for the uniformlyoriented Steiner tree problem. In 10th Annual European Symposium on Algorithms (ESA 2002), volume 2461 of Lecture Notes in Computer Science, pages 760-772. Springer, 2002.

[PWZ04] M. Paluszewski, P. Winter, and M. Zachariasen. A new paradigm for general architecture routing. Proceedings of the 14th ACM Great Lakes Symposium on VLSI (GLSVLSI), pages 202-207, 2004.

[RS98] S.B. Rao and W.D. Smith. Approximating geometric graphs via "spanners" and "banyans". Proceedings of the 30th ACM Symposium on Theory of Computing, pages $540-550,1998$.

[RZ00] G. Robins and A. Zelikovsky. Improved Steiner tree approximation in graphs. Proceedings of the 11th Annual ACM-SIAM Symposium on Discrete Algorithms, pages $770-779,2000$.

[She97] C.F. Shen. The $\lambda$-geometry Steiner minimal tree problem and visualization. $P h D$ thesis, Northwestern University, Evanston, IL, USA, 1997.

[Tei02] S. L. Teig. The X architecture: not your father's diagonal wiring. In SLIP '02: Proceedings of the 2002 international workshop on System-level interconnect prediction, pages 33-37. ACM Press, 2002.

[X] http://www.xinitiative.org.

$\left[\mathrm{ZZJ}^{+} 04\right]$ Q. Zhu, H. Zhou, T. Jing, X. Hong, and Y. Yang. Efficient octilinear Steiner tree construction based on spanning graphs. Proceedings 2004 Asia and South Pacific Design Automation Conference (ASP-DAC), pages 687-690, 2004. 